\title{
En el fondo hablamos la misma lengua (o no): tipologías lingüísticas y universales en la clase de $\mathbf{E} / \mathbf{L E}$
}

CARlos Gallego Martí

Universitat Politècnica de València

cgallego@ade.upv.es

Resumen: El estudio de las tipologías lingüísticas (esto es, de las semejanzas y disimilitudes de las diferentes lenguas presentes en el aula de E/LE con respecto a la lengua española) así como el de los universales (principios comunes a todas las lenguas conocidas del mundo) constituye una herramienta de indudable valor pedagógico puesto que permite a los alumnos una comprensión global del español, de sus límites estructurales y de la particular concepción del mundo que supone comunicarse en este idioma. Asimismo, contribuye a prevenir las transferencias gramaticales equivocadas entre la lengua materna del estudiante y la lengua meta. Finalmente, este enfoque proporciona una visión científica del hecho lingüístico, aspecto que favorece que el nuevo sistema gramatical que el alumno va a aprender sea percibido como un modelo riguroso y coherente, y no como un compendio de normas y relaciones más o menos caprichosas.

Palabras clave: E/LE, tipologías lingüísticas, universales, ejercicios prácticos.

In fact we speak the same language (or not): linguistic and universal typologies in SFL class

Abstract: Studying linguistic typologies (i.e. of similarities and differences between different languages present in the Spanish as a foreign language, SFL class, regarding the Spanish language per se), as well as typologies of universal languages (common principles among languages from all over the world) constitute a tool of undoubted pedagogical value allowing students to achieve a general understanding of Spanish, its structural limits and its particular approach of the world that uses it. In addition, it also contributes to preventing erroneous grammatical transfers between the student's mother tongue and the target language. Finally, this approach provides a scientific view of the linguistic fact, an aspect that favours that the new grammatical system that the student will learn is perceived as a rigorous and coherent system, and not as a compendium of rules and more or less capricious relationships.

Keywords: SFL, linguistic typologies, universal linguistics, practical exercises.

\section{Introducción}

Las tipologías lingüísticas $-\mathrm{y}$, consecuentemente, los universales del lenguajeconstituyen una rama científica que puede resultar de gran ayuda como estrategia metodológica para aplicar en la enseñanza de E/LE. Una clase de estudiantes de español en edad adulta se compone de un grupo heterogéneo en el que confluyen diferentes lenguas, intereses, motivaciones, estilos de aprendizaje y, por supuesto, un nivel desigual de conocimientos lingüísticos. Los alumnos tienen a menudo la sensación de que estudiar un idioma nuevo es tanto como asimilar de manera más o menos explícita (en función del 
enfoque metodológico empleado por el profesor) un sistema gramatical cuya estructura interna difiere completamente de sus lenguas maternas. Paralelamente, la lengua propia se convierte en el punto de partida para la adquisición de la nueva. A causa de este principio de equivalencia (Moreno Cabrera 2015: 81), el discente trasladará de manera inconsciente las estructuras gramaticales desde su lengua materna al sistema lingüístico del español.

El presente trabajo, pensado para un nivel B1, tiene por objetivo mostrar (a través de determinadas actividades basadas en los diferentes niveles de estructuración del lenguaje) que desde la perspectiva de la comparación tipológica de las lenguas los estudiantes pueden calibrar de manera precisa, no intuitiva, la distancia idiomática que los separa del español; por otra parte, contribuye a comprender la lengua de estudio en su globalidad, a captar la «filosofía gramatical o la hechura gramatical de la lengua que está aprendiendo y ello va mucho más allá de un mero conocimiento gramatical concreto y limitado, sino que se extiende hacia todos los recovecos de la lengua objeto de estudio» (Moreno Cabrera $i$ bid.: 81). En efecto, la reflexión sobre las distintas formas de concebir la realidad que supone hablar lenguas diversas coadyuva a la inmersión lingüística y cultural de los estudiantes, que descubren así la particular visión del mundo que subyace tras la lengua española.

Finalmente, este enfoque ofrece a los alumnos una perspectiva científica del hecho lingüístico, aspecto en absoluto baladí. Obviamente, el profesor no puede exigir a los estudiantes nociones amplias y profundas de la ciencia del lenguaje en una clase de E/LE. Sin embargo, sí que puede contribuir a que estos desechen una serie de prejuicios sobre el análisis de la lengua, lo que produce efectos positivos en el aprendizaje, sobre todo cuando la formación intelectual de los discentes se ha gestado en las denominadas ciencias formales o naturales. Se trata, en última instancia, de que comprendan que la ciencia del lenguaje tiene sus propios modelos explicativos y predictivos, rigurosos y estructurados en diferentes niveles de análisis, y que el lenguaje no es una realidad inaprehensible formalmente que haya que abordar desde la una posición intelectual ingenua o poco estructurada.

\section{La clasificación de las lenguas del aula: familias lingüísticas}

En primer lugar, el profesor dispone una actividad para emparentar lingüísticamente las lenguas maternas presentes en el aula -incluyendo, obviamente, el español-. Hay que tener presente que la clasificación lingüística, de acuerdo con Moreno Cabrera (1997: 41 y ss.), puede abordarse desde cuatro criterios distintos.

a) Criterio genético: toma como base el origen y la evolución de las lenguas. En función de este criterio, los diferentes códigos se agrupan en familias (por ejemplo, las lenguas romances) que, a su vez, se pueden vincular en subfamilias (iberorromance) o en macrofamilias (itálica). Por su parte, las macrofamilias pueden formar agrupaciones genéticas mayores llamadas filos (indoeuropeo), macrofilos (euroasiático) y megafilos (nostrático).

b) Criterio geográfico: supone una clasificación de acuerdo con el ámbito geográfico en el que las lenguas se hablan. Puede resultar de una utilidad limitada porque, aunque es frecuente que lenguas que comparten territorio acaben transfiriéndose mutuamente una serie de características, es difícil discernir cuál es la lengua 
dominante que ha influido más sobre la otra $\mathrm{y}$, en consecuencia, en qué direcciones se ha producido esa influencia y por qué (Geenberg 1966; Apud Morena Cabrera op. cit.).

c) Criterio tipológico: desde este parámetro taxonómico se vinculan las lenguas teniendo en cuenta los rasgos gramaticales comunes que presentan. Por supuesto, los rasgos universales no son útiles para establecer una clasificación tipológica, pero sí los aspectos exclusivos de los sistemas lingüísticos. De manera general, nos guiaremos por el parámetro morfosintáctico que, a nuestro juicio, puede ser más ilustrativo para los estudiantes, que no es otro que el del lugar que ocupa el verbo en la oración. Así pues, distinguiremos entre lenguas SVO (sujeto, verbo y objeto) y lenguas SOV (sujeto, objeto y verbo). El hecho de que una lengua pueda incluirse en alguno de estos esquemas (que son, por otra parte, los más habituales para todos los idiomas del mundo) tiene repercusiones en la utilización de categorías gramaticales como las preposiciones, la subordinación relativa o la complementación nominal.

d) Criterio sociolingüístico: de acuerdo con esta clasificación, y partiendo de los criterios clásicos que estableció Stewart, las lenguas presentan un mayor o menor grado de historicidad (desarrollo continuado a lo largo de la historia), estandarización (fijación social de modelos supradialectales), vitalidad (número de hablantes nativos) y homogeneidad (los rasgos que la lengua presenta en sincronía se han heredado históricamente o derivan del contacto con otros sistemas lingüísticos, como ocurre con los pidgin o con las lenguas criollas).

En nuestro caso, clasificaremos las lenguas del aula atendiendo al primero de los enfoques ${ }^{1}$. Para empezar, elaboramos un listado de las lenguas maternas de los alumnos en la que incluimos, obviamente, el español. Les pedimos que las agrupen de la manera que consideren más apropiada. Con toda probabilidad, los estudiantes escogerán espontáneamente el criterio genético. Leídas las diferentes propuestas taxonómicas que surjan, el profesor muestra la respuesta ${ }^{2}$.

\footnotetext{
${ }^{1}$ Para la vinculación genética de las lenguas que nos podamos encontrar presentes en el aula, véase López García (1994: 18 y ss.). La experiencia práctica que incluyo en el artículo está realizada en una clase de B1 en el marco del programa Erasmus, por lo que mayoritariamente las lenguas maternas de los estudiantes comparten el filo indoeuropeo.

${ }^{2}$ El objetivo de esta sencilla actividad, así como el de todas las que presentamos en este trabajo, no es otro que el de propiciar la reflexión lingüística en el ámbito de las tipologías y de los universales con el fin de situar al alumno, como decíamos al inicio, ante la globalidad y la filosofía de la lengua española. Subsidiariamente, claro está, estamos también trabajando en cada una de ellas cuestiones de léxico, morfosintaxis e incluso aspectos pragmáticos y culturales.
} 


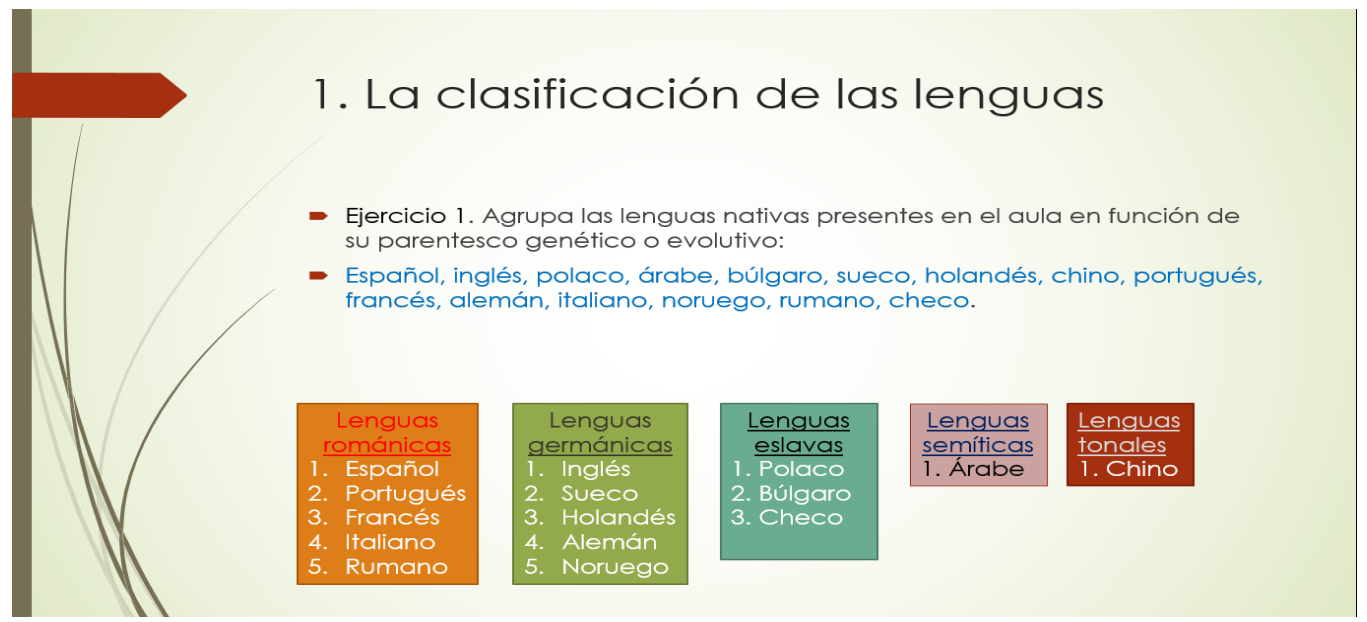

Imagen 1. Clasificación de las lenguas del aula.

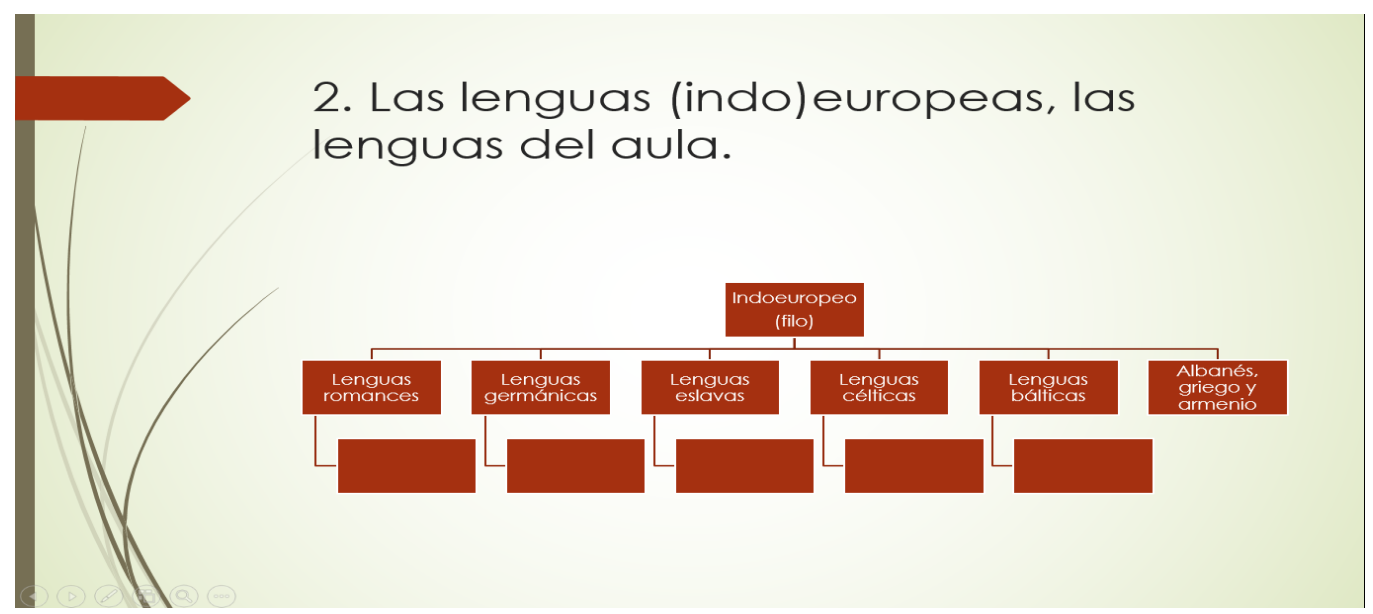

Imagen 2. El filo indoeuropeo.

\section{Criterio tipológico: el alineamiento morfosintáctico del español.}

Desde el plano de análisis morfosintáctico, los alumnos pueden situar la lengua española dentro de un patrón de configuración oracional que observa el lugar que ocupa el verbo y los papeles semánticos principales exigidos por este (sujeto y objeto), y compararlo con el que presentan habitualmente sus lenguas maternas. En esta disposición, universalmente predominan las combinaciones VSO, SOV y SVO, presentes en casi el $90 \%$ de las lenguas del mundo (Moreno Cabrera 1997: 73). La diferencia fundamental entre estos tres parámetros básicos y el que puedan presentar otros códigos radica en que, para estos tipos mayoritarios, el sujeto precede al objeto; solo en unas pocas lenguas, apenas un $4 \%$ según el trabajo de Tomlin ${ }^{3}$ (1986: 22), el objeto precede al sujeto. El hecho de que un sistema lingüístico manifieste uno $\mathrm{u}$ otro esquema oracional tiene repercusiones

\footnotetext{
${ }^{3} \mathrm{R}$. Tomlin estudia el orden de las palabras en la oración sobre la base de un corpus de 1063 lenguas. Sobre el total de ese corpus, son 33 las lenguas en las que el objeto antecede al sujeto.
} 
morfosintácticas de diferente calado. W. Lehmann (1973: 47-66; Apud Moreno Cabrera 2015: 78) las sintetizaba así para el caso de lenguas SVO y SOV.

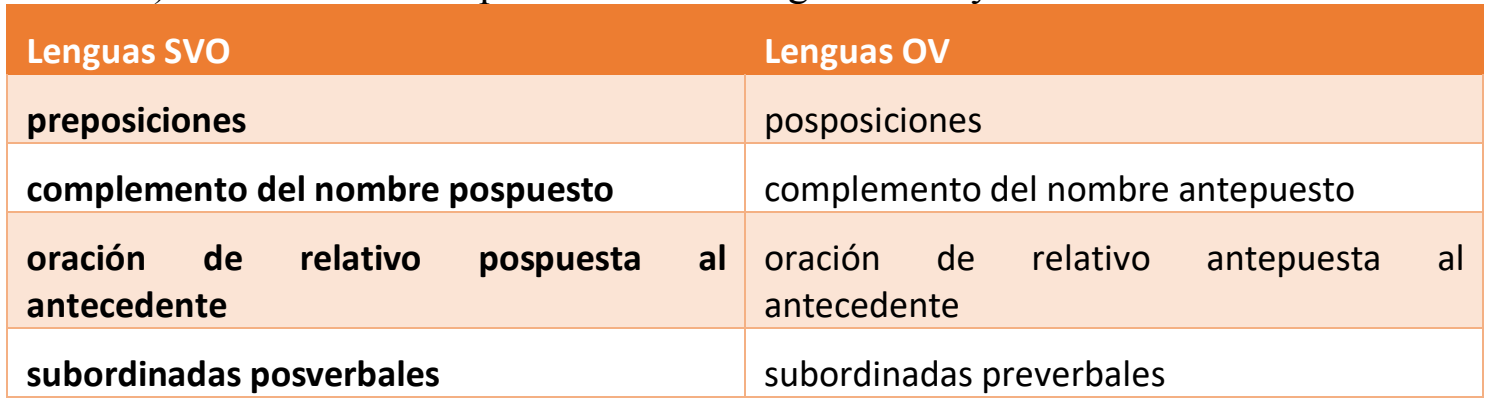

Imagen 3. Implicaciones sintácticas por tipología lingüística.

La siguiente actividad propone una reflexión en el ámbito del alineamiento morfosintáctico que venimos comentando. El profesor muestra en primer lugar una oración sencilla en español, y pide a los alumnos que identifiquen el verbo, además de los tipos argumentales de sujeto y objeto. A continuación, se les solicita que escriban la oración en su lengua materna y que identifiquen esas mismas funciones. Es probable que se produzca una coincidencia tipológica, sobre todo si la lengua dominante del alumno pertenece al filo indoeuropeo. Por último, el profesor explica dos casos ${ }^{4}$-euskera y árabeque presentan un parámetro morfosintáctico diferente (SOV y VSO, respectivamente). En la segunda parte del ejercicio se observan las repercusiones sintácticas de la tipología dominante en español ${ }^{5}$ con algunos ejemplos (lugar que ocupan las preposiciones, los complementos del nombre, las subordinadas de relativo y las subordinadas sustantivas). Las oraciones marcadas como agramaticales representan cómo serían las equivalencias en una lengua de diferente alineamiento morfosintáctico.

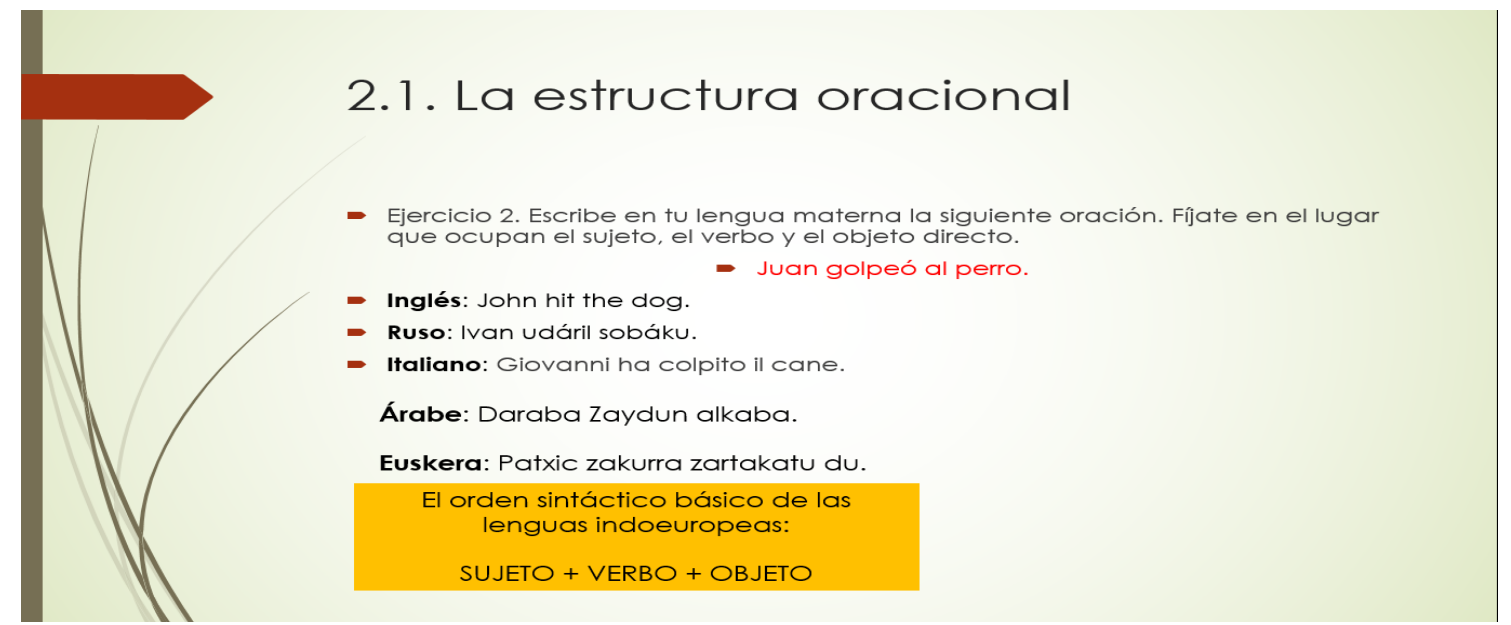

Imagen 4. Orden sintáctico predominante en las lenguas indoeuropeas.

\footnotetext{
${ }^{4}$ Son las lenguas que se escogieron para presentar la experiencia práctica en el aula. Por supuesto, el profesor puede emplear otras distintas siempre y cuando presenten diferentes parámetros morfosintácticos.

${ }^{5}$ Evidentemente, las posibilidades de combinación oracional del español y de cualquier lengua no se agotan en uno de estos esquemas. Todos los idiomas tienen mecanismos para producir alteraciones argumentales en función de los distintos propósitos comunicativos y posibilidades expresivas. Hablamos, en todo caso, del esquema sintáctico que se considera no marcado, esto es, extensivo, por defecto.
} 


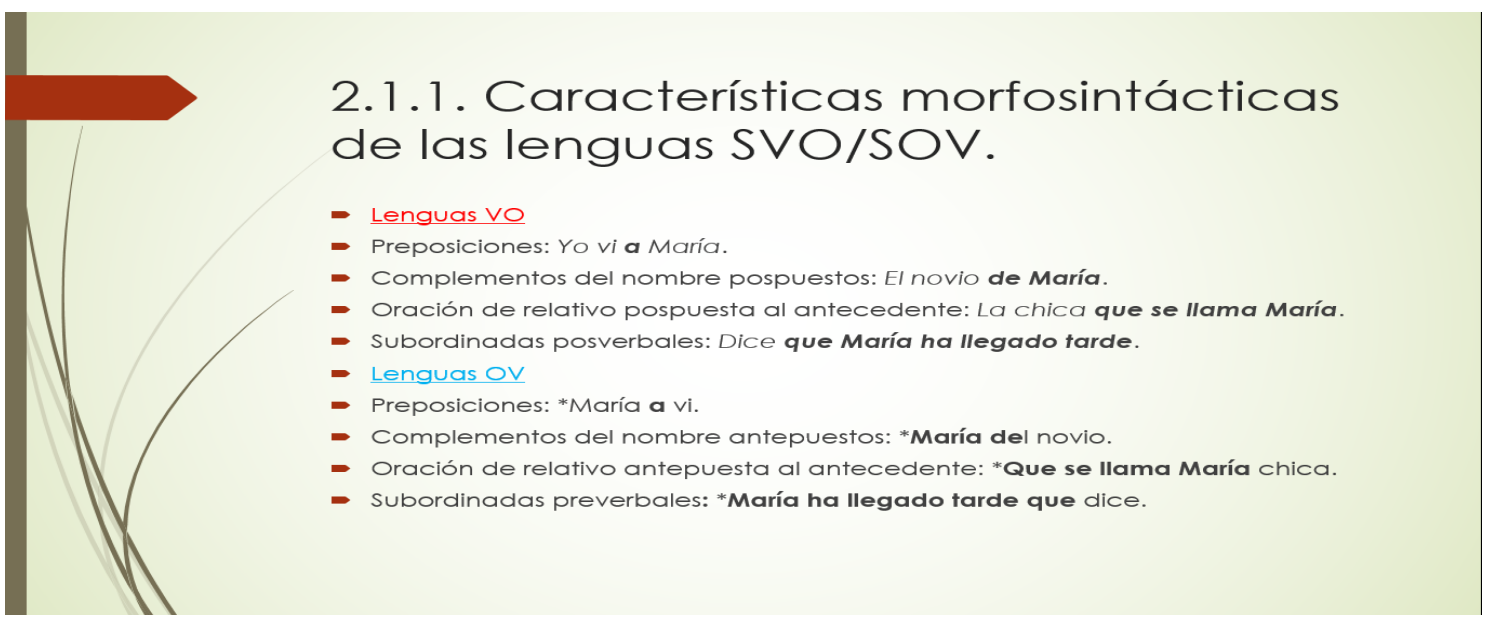

Imagen 5. Comparación del comportamiento sintáctico en lenguas $\mathrm{VO} / \mathrm{OV}$.

\section{La configuración lingüística de la realidad: el vocabulario.}

Las lenguas estructuran y parcelan la visión que los hablantes tienen del mundo de acuerdo con sus características formales. En el plano semántico, los inventarios de elementos léxicos o vocabularios permiten la aprehensión del mundo extralingüístico. Dicho de otro modo, hablar una lengua es concebir la realidad de determinada manera. En clase de E/LE, dadas las características, intereses y objetivos de los alumnos -y del propio proceso de enseñanza-aprendizaje-, no conviene adentrarse en discusiones lingüísticas densas y espinosas. ¿Son las lenguas representaciones simbólicas del pensamiento (teorías experiencialistas ${ }^{6}$ ) o, por el contrario, constituyen abstracciones directas de la realidad y son independientes de aquel (teorías objetivistas)? Por otra parte, ¿es el lenguaje el que parcela el mundo y lo mediatiza, en la línea de las propuestas clásicas de Wittgenstein, o no hay realidad posible fuera del lenguaje, de acuerdo con los postulados de Humboldt? Sea como fuere, lo que parece indudable es que «la propia forma de cada una de las lenguas condiciona la visión que los hablantes desarrollan sobre los objetos del mundo una vez referenciados» (Calvo 1994: 206) ${ }^{7}$.

El ejercicio que presentamos a continuación aborda el estudio del léxico desde una doble perspectiva: de un lado, los alumnos descubren que los vocabularios de sus respectivas lenguas no son tan distintos como en principio cabría esperar puesto que tienen un origen común (indoeuropeo). Del otro, permite una reflexión en la dirección que apuntábamos más arriba: detrás de sistema de la legua se esconde una manera particular de concebir el mundo. En la primera parte de la actividad el profesor pide a los estudiantes que escriban en su lengua materna algunas palabras (en el ejemplo propuesto, madre, uno, dos, tres, noche y mente). Comparando los resultados con las voces españolas se observa una raíz

\footnotetext{
${ }^{6}$ Para profundizar sobre la relación entre lenguaje y pensamiento, véase Cuenca, Ma . J. y Hilferty, J. (2007): Introducción a la lingüística cognitiva. Barcelona, Ariel, p. 17 y ss. Sobre este particular, coincidimos con Moreno Cabrera (1997: 242) cuando señala que las estructuras lingüísticas están -materialmentemotivadas, pero que, una vez creadas, son independientes de esa base material o cognitiva.

${ }^{7}$ En López García et alii (1994): Lingüistica general y aplicada. Valencia, Servei de Publicacions de la Universitat de València.
} 
común, y, por lo tanto, un mismo origen que permite agrupar las lenguas del aula en el filo indoeuropeo. Del mismo modo, el significado de las palabras propuestas no diferirá de una lengua a otra. En la segunda parte de la actividad, proponemos estudiar la etimología de la palabra «trabajo». La elección del término, lejos de ser aleatoria, permite adentrarse en aspectos culturales, concretamente en el mundo de los clichés y de los estereotipos. Resulta interesante, en este sentido, preguntar a los discentes sobre las ideas preconcebidas que tienen sobre los horarios laborales de España y, en general, sobre la actitud hacia el mundo del trabajo de los hispanohablantes. «Trabajar» deriva del latín vulgar *tripaliāre y este, a su vez, del latín tardío tripalium 'instrumento de tortura compuesto de tres maderos'. Etimológicamente, por lo tanto, el trabajo es un sufrimiento. Como decíamos anteriormente, es evidente que la parcelación de la realidad que efectúan los vocabularios de cada lengua manifiesta en mayor o menor grado una concepción del mundo. Sin embargo, y el actual significado del sustantivo «trabajo» es una buena prueba de ello, una vez ahormada esa realidad o concepto mediante la palabra, esta adquiere independencia. Dicho de otro modo, lengua y pensamiento -o lengua y mundo- son dos momentos del mismo proceso intelectivo de aprehensión de la realidad, pero no son la misma cosa.

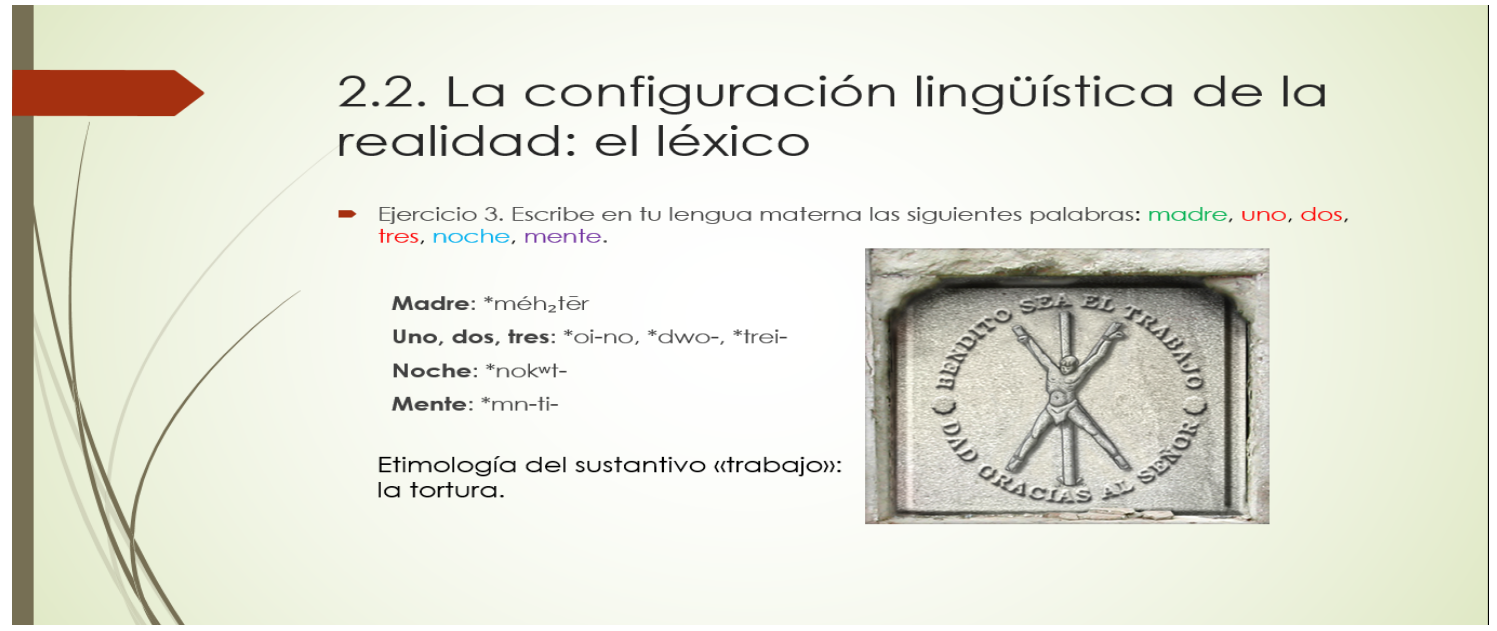

Imagen 6. Cognados: léxico de origen indoeuropeo.

\section{Los universales lingüísticos: la morfología y el léxico}

Los universales lingüísticos son todos aquellos «rasgos o propiedades que son comunes a todas las lenguas humanas presentes, pasadas y futuras y que, por tanto, configuran lo que se puede concebir como el conjunto de características o propiedades que definen al lenguaje humano en general» (Moreno Cabrera 1997: 53). De entre los múltiples aspectos que podríamos destacar desde esta perspectiva (universales semióticos y gramaticales, universales fonológicos, jerarquías, procesos de gramaticalización, etc.), planteamos llevar al aula dos propuestas didácticas enfocadas en los niveles morfológico y léxico. En los epígrafes anteriores el profesor ha conseguido mostrar a los alumnos el esqueleto tipológico del español y su relación con otras lenguas de su entorno. Ahora se centrará en los aspectos coincidentes de todos los idiomas naturales 
$\operatorname{conocidos}^{8}$. Desde una perspectiva amplia, más allá del parentesco genético del español con las lenguas presentes en la clase, todos y cada uno de los sistemas lingüísticos están relacionados por una serie de rasgos comunes (lo que parecería apuntar a un origen biológico, innato, del lenguaje, en la línea de las ideas chosmskyanas).

La primera tarea consiste en ubicar al español dentro de la jerarquía universal morfológica como lengua flexiva. En primer lugar se invita a reflexionar sobre las posibilidades morfológicas del español para ampliar el inventario léxico a partir de elementos ya existentes (flexión, composición y derivación).

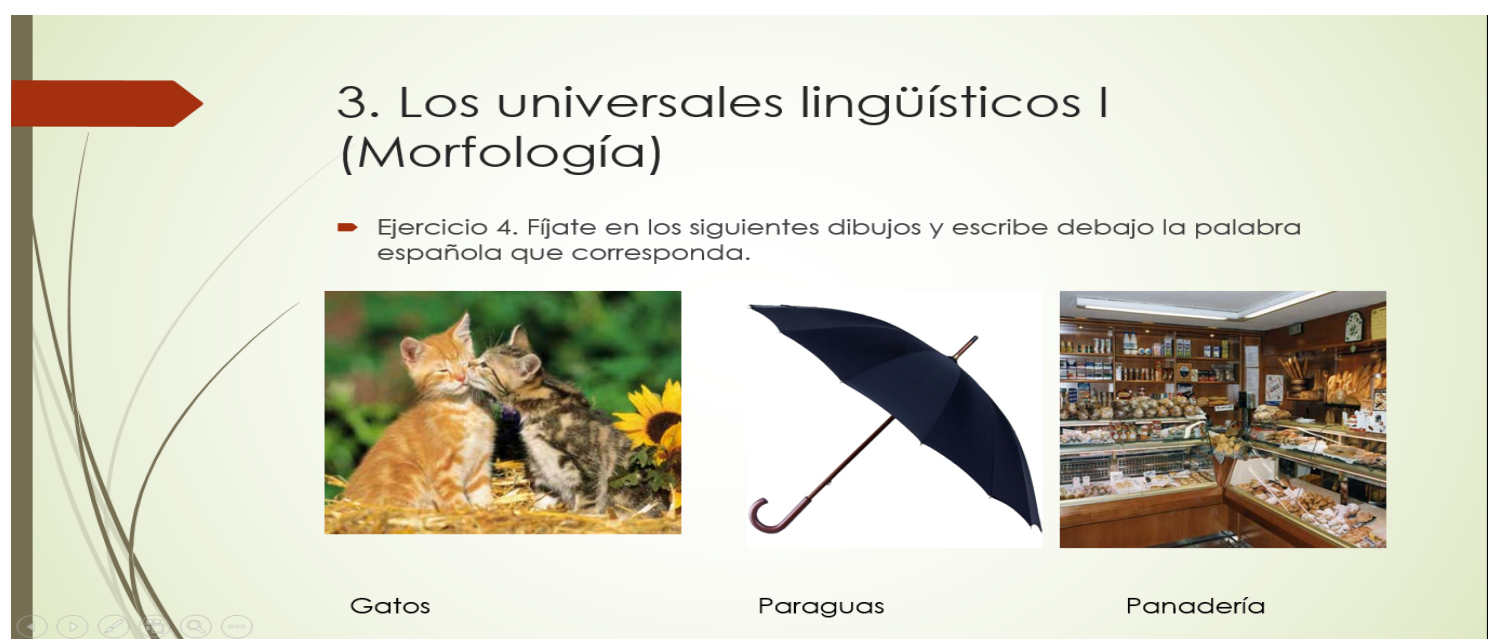

Imagen 7. Flexión, composición y derivación en español.

Posteriormente, tras comparar el caso del español con la lengua materna del alumno, se muestra la jerarquía universal de la formación de palabras: si una lengua conoce la flexión, conocerá la derivación; del mismo modo, si tiene prefijos y sufijos, podrá formar palabras compuestas. De manera inversa, no todas las lenguas que conocen la derivación compositiva tienen afijos; finalmente, aunque una lengua tenga afijos puede que no desarrolle una morfología flexiva. El español, por lo tanto, es una lengua flexiva porque amalgama y fusiona la información gramatical tras la raíz, pero no aglutinante (un morfema representa un solo significado gramatical) ni aislante (carecen de morfología flexiva nominal y verbal).

8 Téngase en cuenta, como apunta Moreno Cabrera, que un universal lingüístico es siempre una característica provisional que se puede mantener en tanto en cuanto no se descubra en el futuro una lengua natural que no presente dicho rasgo. En este sentido, se trata siempre de una propuesta dinámica, sujeta a revisión. 


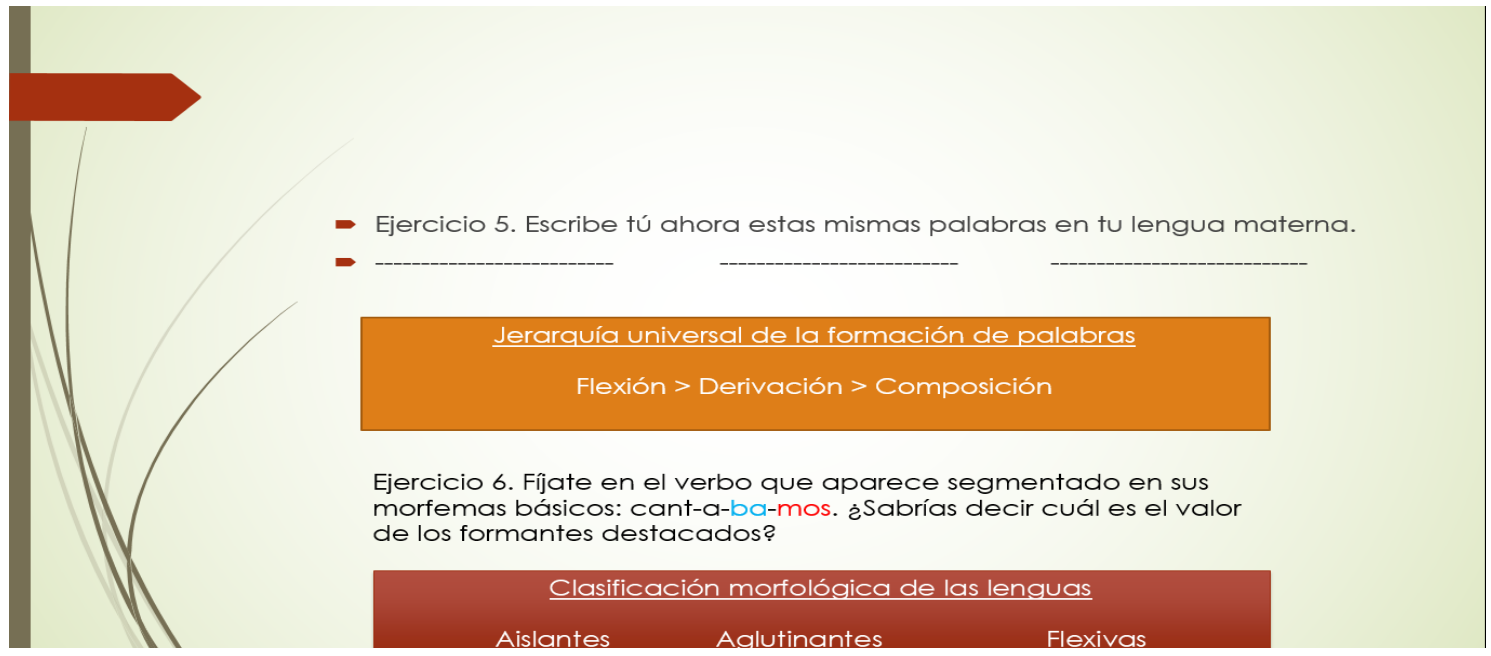

Imagen 8. Lenguas aislantes, aglutinantes y flexivas.

La última propuesta didáctica consiste en presentar para el caso del español la jerarquía universal de la abstracción metafórica con la pretensión de que los alumnos busquen ejemplos análogos en sus propias lenguas y de que perciban, en última instancia, cómo funciona el universal semántico de la lexicalización, proceso por el que un elemento gramatical, morfosintáctico, pasa a engrosar el inventario del vocabulario de una lengua: correveidile, metomentodo, pordiosero...
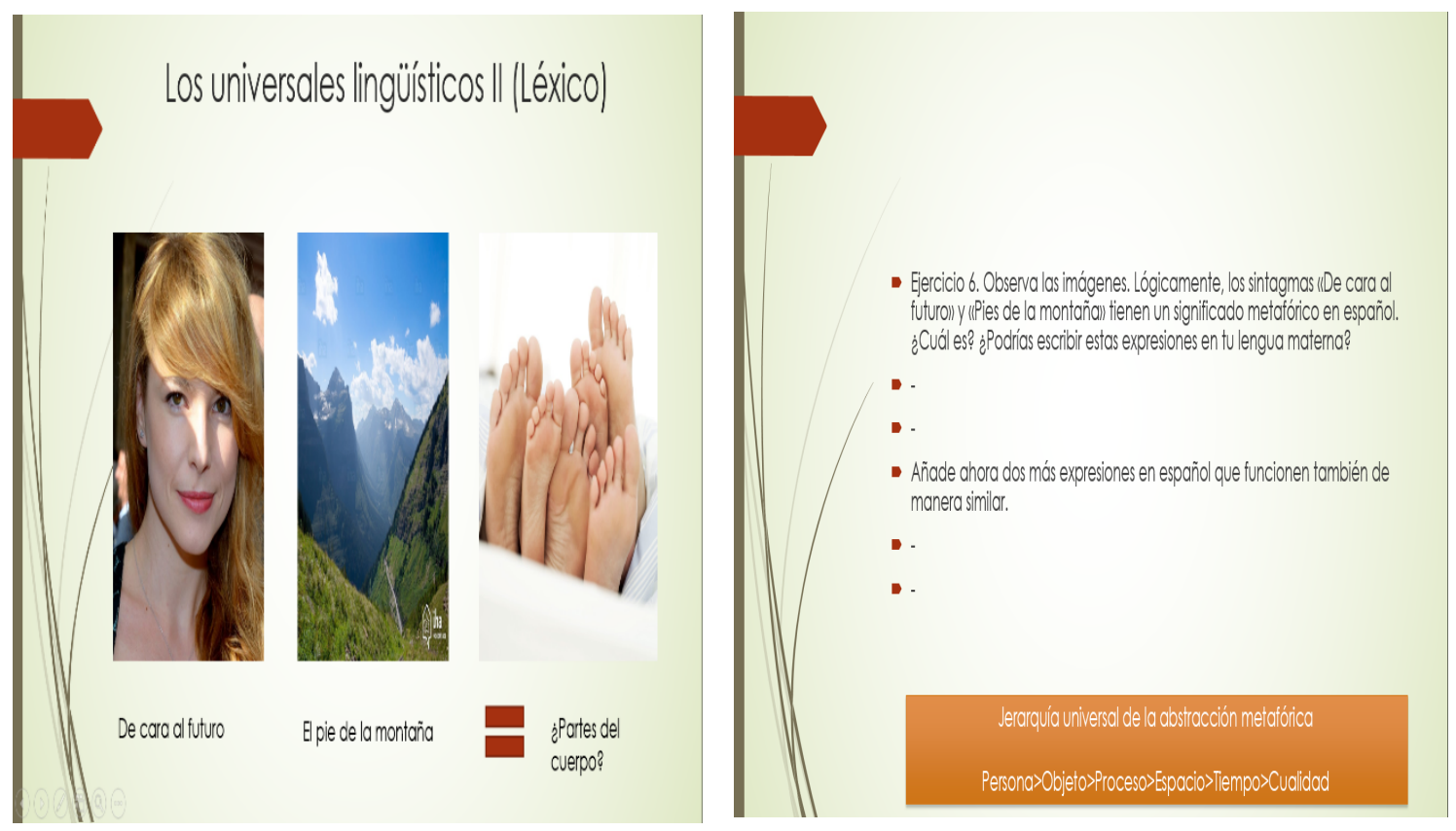

Imagen 9. Jerarquía universal de la abstracción metafórica. 


\section{Conclusión}

El estudio del español como lengua meta, independientemente del enfoque metodológico utilizado en clase, puede enriquecerse extraordinariamente con las aportaciones provenientes de la lingüística comparada. A lo largo de este trabajo hemos pretendido mostrar una propuesta práctica para acercar a los alumnos de E/LE una perspectiva del idioma basada en las tipologías lingüísticas y en los universales. Pensamos que esta aproximación contribuye poderosamente a perfilar en toda su dimensión la estructura gramatical de la lengua española en sus distintos niveles de análisis. A través de ella, los estudiantes descubren las similitudes y diferencias que existen entre las lenguas del aula y adquieren una base de conocimientos que hace más coherente el aprendizaje de la gramática del español. Hablar cualquier lengua implica concebir la realidad sensible o imaginada de determinada manera; los diferentes parámetros de variación tipológica dan cuenta de este hecho de manera diáfana. Por último, una experiencia didáctica de este cariz muestra a los ojos de los discentes (a menudo, con escasa formación en este campo) que la lingüística ocupa un lugar dentro del análisis científico, con sus propios modelos y métodos, y que nada en la conformación del lenguaje es casual, ingenuo o desestructurado.

\section{Bibliografía}

CuencA, Maria Josep y Hilferty, Joseph. (2007). Introducción a la lingüística cognitiva. Barcelona: Ariel.

ESCANDELl VidAL, M. ${ }^{a}$ Victoria. (2004). Fundamentos de semántica composicional. Barcelona: Ariel.

Instituto Cervantes (2006). Plan curricular del instituto Cervantes. Madrid: Biblioteca Nueva.

LEHMANN, Winfred. P. (1973). «A structural principle of langauge and its implications». Lenguage, 49, 47-66.

LÓPEZ GARCÍA, Ángel (et alii) (1994). Lingüística general y aplicada. Valencia: Universitat de València.

Ministerio DE EDUCACIÓN, CULTURA Y DEPORTE (2002). Marco común europeo de referencia para las lenguas: aprendizaje, enseñanza y evaluación. Madrid: MECDAnaya (coedit.).

MORENO CABRERA, Juan Carlos (1997): Introducción a la lingüística. Enfoque tipológico y universalista. Madrid: Síntesis.

Moreno CABrera, Juan Carlos (2015). «¿Cómo puede ayudar la tipología lingüística a la didáctica de ELE?». En Yuko Marimoto, María Victoria Pavón y Rocío Santamaría (eds.), La enseñanza de ELE centrada en el alumno (pp. 77-85). Getafe: Asociación para la enseñanza del español como lengua extranjera. https://dialnet.unirioja.es/servlet/articulo?codigo $=5425851$

Tomlin, Rusell. S. (1886). Basic word order. Functional principles. Londres: Croom Helm. 